\title{
A Case of Hepatocellular Carcinoma with Portal Vein Tumor Thrombosis Treated by Hepatic Arterial Infusion Chemotherapy and Radiotherapy
}

\author{
Jin Yong Lee ${ }^{1 *}$, Jeong-Ju Yoo ${ }^{* *}$, Seong Joon Chun', Sun Hyun Bae ${ }^{2}$, Jae Myeong Lee ${ }^{3}$, Sang Gyune Kim, \\ Young Seok $\mathrm{Kim}^{1}$ \\ 'Division of Gastroenterology and Hepatology, Department of Internal Medicine, ${ }^{2}$ Department of Radiology and Research Institute of \\ Radiology, ${ }^{3}$ Department of Radiation Oncology, Soonchunhyang University Bucheon Hospital, Soonchunhyang University School of \\ Medicine, Bucheon, Korea
}

Received Dec. 23, 2019

Revised Jan. 29, 2020

Accepted Feb. 19, 2020
Sorafenib is the standard treatment for advanced hepatocellular carcinoma (HCC) with portal vein thrombosis (PVT). Additionally, hepatic arterial infusion chemotherapy has been used as a treatment option for advanced HCC. Here, we report a case of sustained partial response in a patient with advanced HCC with PVT after hepatic arterial infusion chemotherapy and radiation therapy. (J Liver Cancer 2020;20:78-83)

Keywords: Hepatocellular carcinoma; Chemotherapy; Radiotherapy

\section{INTRODUCTION}

Hepatocellular carcinoma (HCC) is the fifth most common cancer worldwide, and an estimated 650,000 people die from HCC per year. ${ }^{1}$ Especially, advanced HCC shows a poor prognosis despite multiple aggressive treatment options. Moreover, in the case of main portal vein thrombosis (PVT), the 5-year survival rate was reported as only $4.6 \%{ }^{2}$ According to the treatment guidelines, sorafenib is the standard treatment option, and external beam radiation therapy (EBRT) and transarterial chemoembolization (TACE) can be used as alternative treatments. ${ }^{3,4}$ Although several studies have reported the efficacy of hepatic arterial infusion chemo-

\section{Corresponding author : Young Seok Kim}

Division of Gastroenterology and Hepatology, Department of Internal Medicine, Soonchunhyang University Bucheon Hospital, 170 Jomaru-ro, Wonmi-gu, Bucheon 14584, Korea

Tel. +82-32-621-5079, Fax. +82-32-621-5018

E-mail; liverkys@schmc.ac.kr

https://orcid.org/0000-0002-7113-3623 therapy (HAIC) in the treatment of advanced HCC, the guidelines do not recommend HAIC as a standard treatment. Therefore, HAIC may be a potential treatment option for advanced HCC, which could be beneficial to those patients who cannot tolerate tyrosine kinase inhibitors. ${ }^{5,6}$ Here, we report the case of a sustained partial response in a patient with advanced HCC with PVT after HAIC and radiation therapy.

\section{CASE REPORT}

A 64-year-old man was admitted to the hospital with hematemesis. Previously, he was diagnosed with hepatitis B but did not undergo regular surveillance or treatment. He did not drink alcohol. He was diagnosed with diabetes and was taking insulin. At the time of admission, the patient's blood pressure and pulse rate were 110/70 $\mathrm{mmHg}$ and 98 beats/ minutes, respectively. Laboratory findings of peripheral blood tests showed white blood cells $16,450 / \mathrm{mm}^{3}$ (neutrophils $86.1 \%$ and eosinophils $0.1 \%$ ), hemoglobin $10.9 \mathrm{~g} / \mathrm{dL}$,

\footnotetext{
* The first two authors contributed equally to this study.
} 
and platelets $255,000 / \mathrm{mm}^{3}$. The biochemistry tests showed total protein $6.5 \mathrm{~g} / \mathrm{dL}$, albumin $3.3 \mathrm{~g} / \mathrm{dL}$, fasting blood sugar $483 \mathrm{mg} / \mathrm{dL}$, blood urea nitrogen $25.9 \mathrm{mg} / \mathrm{dL}$, creatinine $1.5 \mathrm{mg} / \mathrm{dL}$, aspartate aminotransferase/alanine aminotransferase $65 / 50 \mathrm{U} / \mathrm{L}$, alkaline phosphatase $229 \mathrm{U} / \mathrm{L}$, total cholesterol $148 \mathrm{mg} / \mathrm{dL}$, total bilirubin $1.52 \mathrm{mg} / \mathrm{dL}$ (direct bilirubin $0.65 \mathrm{mg} / \mathrm{dL}$ ), and prothrombin time 13.9 seconds (international normalized ratio 1.20). The viral marker test results were hepatitis B surface antigen (HBsAg) (+), anti-HBs Ab $(-)$, anti-hepatitis $\mathrm{C} \mathrm{Ab}(-)$, HBe antigen/anti-HBe antibody $(-/+)$, antibody to the hepatitis B core antigen (IgG anti$\mathrm{HBc})(+)$, and HBV DNA $<15 \mathrm{IU} / \mathrm{mL}$. Alpha-fetoprotein (AFP) was $5,203 \mathrm{ng} / \mathrm{mL}$, and the concentration of protein induced by vitamin K absence-II (PIVKA-II) was 3,183 mAU/ $\mathrm{mL}$. The patient had preserved liver function with Child-
Pugh class $\mathrm{A}^{6}$ and a model for end stage liver disease score of 8. The performance status was grade 1 according to the Criteria of the Eastern Cooperative Oncology Group.

An emergency gastroscopy was performed. Esophageal variceal bleeding was found, and the patient underwent endoscopic variceal ligation. The patient was stabilized after variceal ligation, and additional imaging tests were performed.

Dynamic contrast-enhanced computed tomography (CT) revealed mass lesions in segment 5/6. The mass had an irregular margin and showed heterogeneous enhancement in the arterial phase and iso-attenuation in segment 5/6 in the portal venous phase. In addition to the main mass, multiple thromboses of the main portal vein (PV), right PV (RPV), and left PV (LPV) umbilical segments were found (Fig. 1).
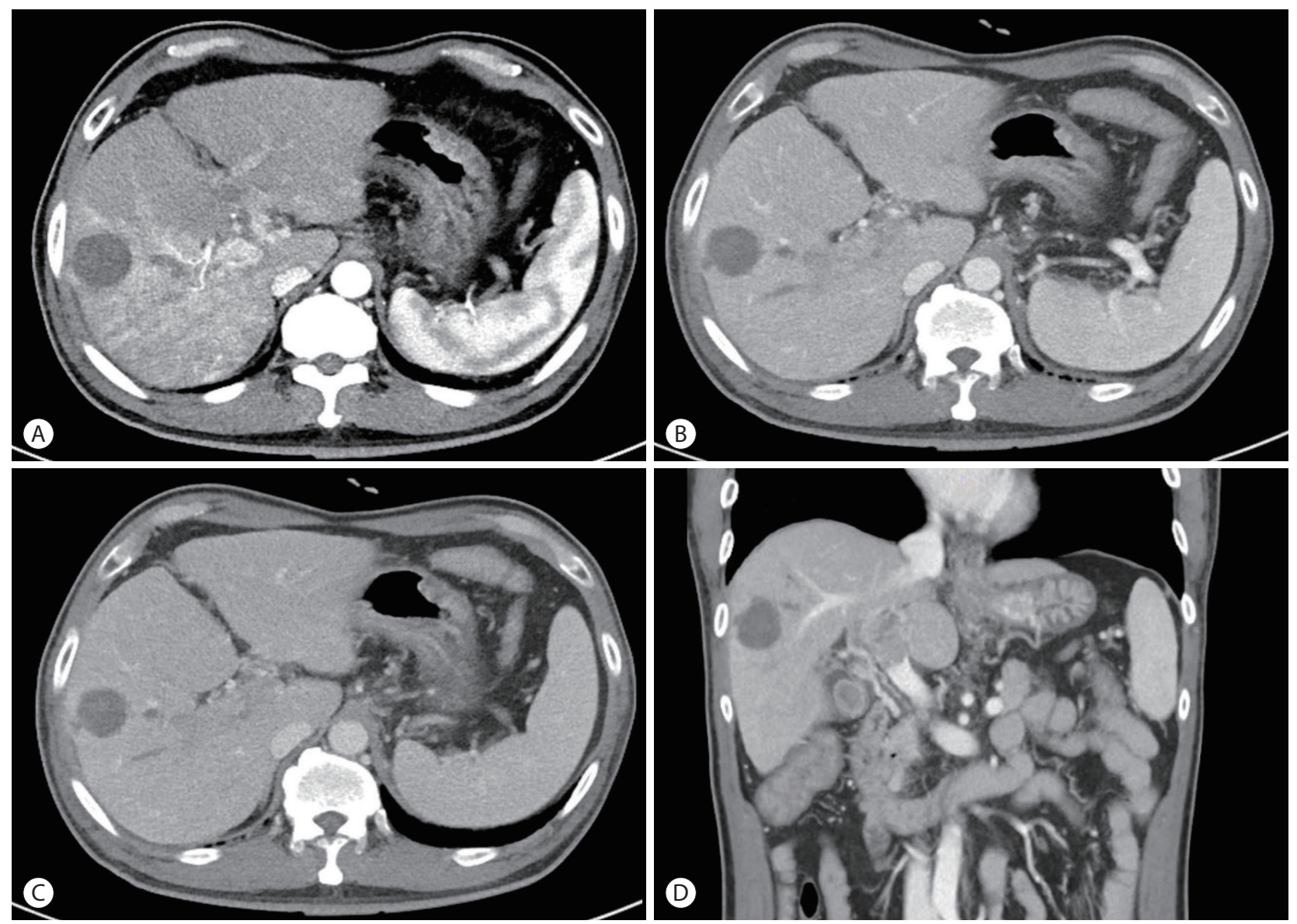

Figure 1. Baseline computed tomography imaging. In segment 5/6, a round heterogeneous enhanced mass with an irregular margin was observed in the (A) arterial phase followed by iso-attenuation in the (B) portal phase and the (C) venous phase. In contrast, the portal vein (PV) thrombus in the right hepatic vein was slightly hypointense (C). PV tumor thrombosis was enhanced in the main PV (D). 
The magnetic resonance image (MRI) showed a round lesion on segment 5 with a slightly high density on the non-enhanced scan, suggesting it may be an infarcted mass. In the arterial phase, the right lobe of the liver was enhanced followed by a suspected washout in the portal delayed phase. The thrombi inside the main PV, RPV, and LPV umbilical segments showed a similar enhancement pattern. The T2 phase image showed irregular high-signal mass lesions spread in the right lobe. The right anterior segment of the liver was nearly replaced by the tumor. A 20-minutes delayed image showed lesions with signal defects (Fig. 2). Finally, the mass was diagnosed as infiltrative advanced HCC with PVT. The stage of HCC was IVa in the modified Union for International Cancer Control stage and stage $\mathrm{C}$ according to the
Barcelona Clinic Liver Cancer stage system.

The patient underwent a combination of HAIC and radiation therapy. HAIC was administered in fixed doses of $500 \mathrm{mg}$ of 5-fluorouracil for three days and $60 \mathrm{mg}$ of cisplatin for one day at four-week intervals. Radiation therapy of 45 Gy was administered in 25 fractions. To reduce the risk of radiationinduced liver injury, radiation therapy was administered only to the PVT. However, hematemesis caused by duodenal ulcer occurred after the 5 th day of radiation treatment. Subsequently, only HAIC without radiotherapy was maintained. After six HAIC treatments at four-week intervals, CT and MRI showed a marked decrease in the size of the previously invasive lesions. In addition, the size of the extensive PVT decreased (Fig. 3). Based on the CT results, right hepatecto-
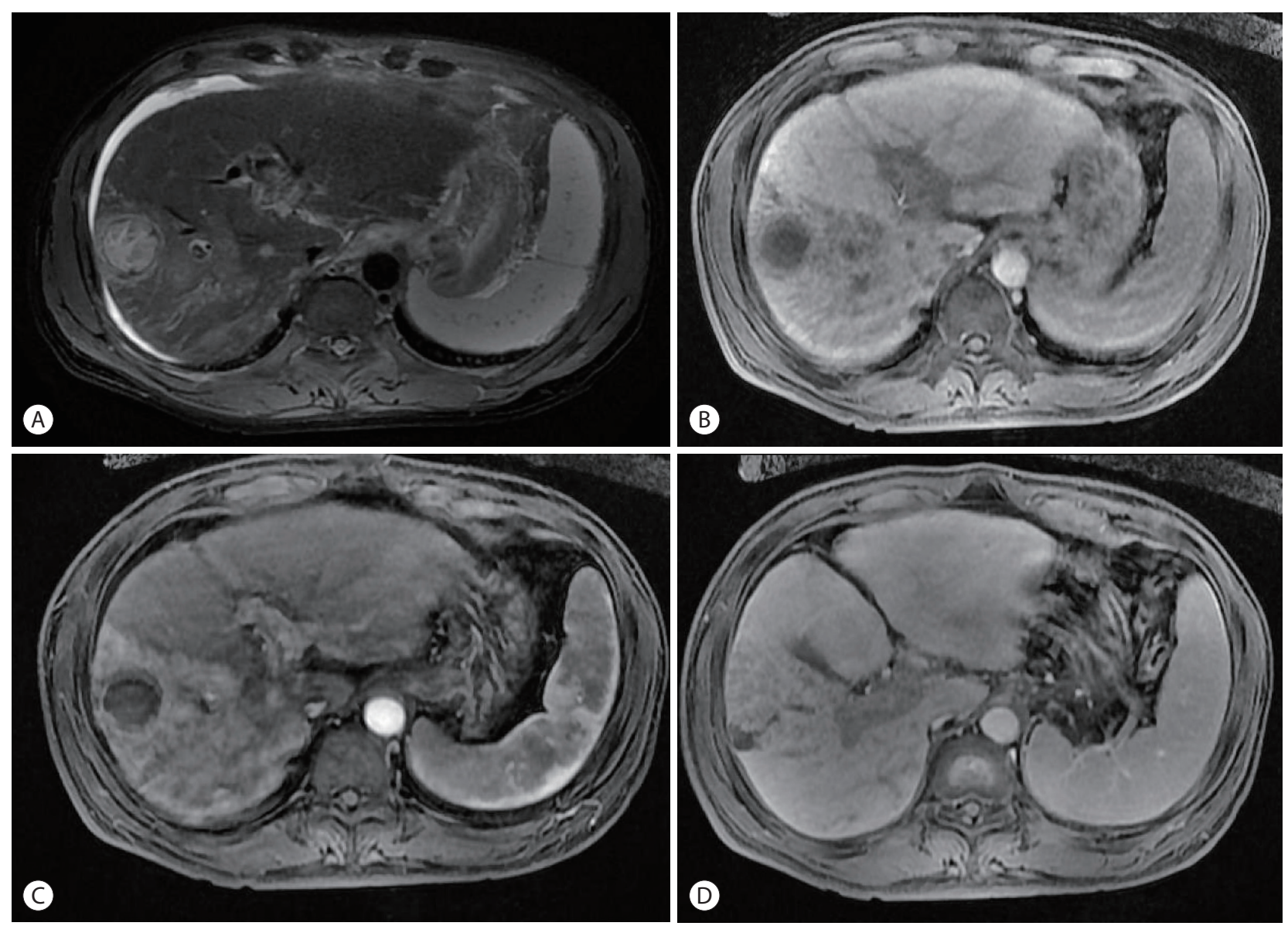

Figure 2. Baseline magnetic resonance imaging (MRI). Liver MRI findings: infiltrative hepatocellular carcinoma was observed in the right hepatic lobe with a tumor nodule in segment $5 / 6$. The lesion was ill-defined with high attenuation in the right hepatic lobe in (A) the arterial phase followed by wash-out in the portal delayed and portal venous phase $(B, C)$. In addition, a portal vein tumor thrombus was observed in the hepatobiliary phase (D). 
my was considered, but the operation was cancelled due to three-vessel coronary disease. Instead of resection, TACE was performed. Follow-up imaging was performed 27 months after the first HAIC treatment and 16 months after TACE. The last follow-up image showed a stable state with a considerably regressed infiltrative HCC in the right lobe, infarcted HCC at segment 5/6, and decreased PVT in both portal veins (Fig. 4). During the treatment, AFP decreased from 5,203 ng/ $\mathrm{mL}$ to $3.4 \mathrm{ng} / \mathrm{mL}$ and PIVKA-II decreased from 3,183 AU/ $\mathrm{mL}$ to $19 \mathrm{mAU} / \mathrm{mL}$.

Informed consent was waived because of the retrospective nature of the study and anonymous clinical data. This study was approved by the Institutional Review Board of Soonchunhyang University Hospital, Bucheon, Korea (SCHBC 2019-11-007). The study protocol conformed to the ethical guidelines of the World Medical Association Declaration of Helsinki.

\section{DISCUSSION}

HCC has a high mortality rate of $14.7 \%$ which is the second-highest after lung cancer. ${ }^{7}$ Recent advances in diagnostic methods have enabled early detection and diagnosis, which have greatly improved the outcome of HCC treatment. However, the prognosis of advanced HCC has not improved despite various treatment options. In Korea, advanced HCC with PVT is common, accounting for about $30 \%$ of the newly diagnosed HCC patients. In this advanced stage, sorafenib is the standard treatment option for patients with preserved liver function. ${ }^{3,4}$ However, the efficacy of sorafenib is not suf-
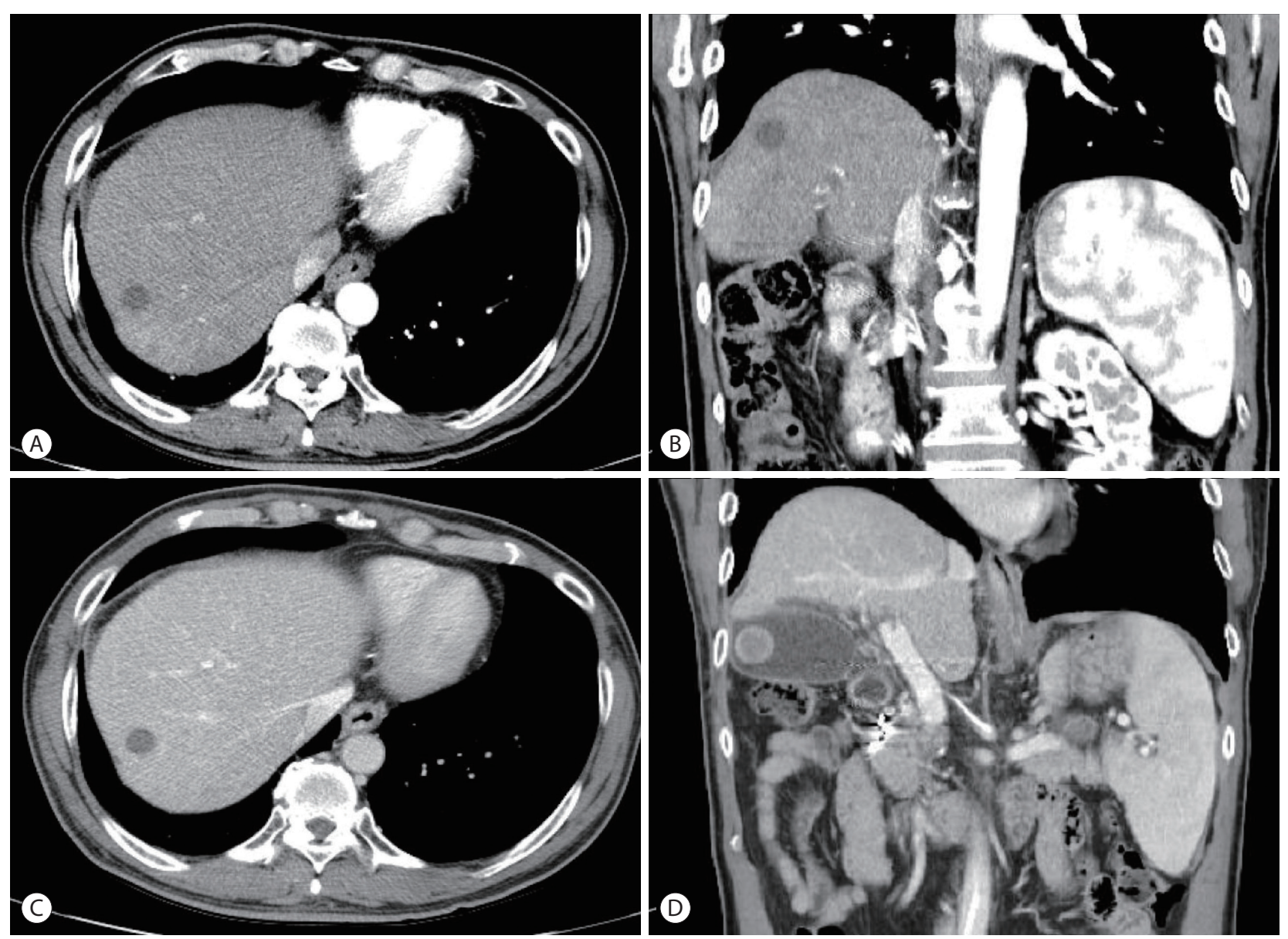

Figure 3. Follow-up computed tomography (CT) finding after six months. CT and showed marked improvement in the infiltrative hepatocellular carcinoma (HCC) in the right lobe with a decrease in the size of the infarcted HCC in segment 5/6 (A-C). In addition, the size of the extensive portal vein thrombosis decreased in the main vein and both portal veins $(D)$. 
ficient. Therefore, various combination therapies or other treatment options such as HAIC, TACE, and EBRT have been used to overcome the insufficient efficacy of sorafenib. Although these are not standard treatment modalities, they have demonstrated desirable therapeutic efficacy in selected patients. Among them, HAIC was shown to be effective in recent studies. A randomized, comparative study between sorafenib and HAIC was performed in 58 patients with advanced HCC with PVT. ${ }^{8}$ The median overall survival (OS) (14.9 vs.7.2 months) and time to progression (TTP) (4.4 vs. 2.7 months) were significantly longer in the HAIC group than in the sorafenib group. Moreover, Song et al. ${ }^{5}$ reported that the OS (HAIC vs. sorafenib: 7.1 vs. 5.5 months) and TTP of HAIC was comparable to that of sorafenib in ad- vanced HCC patients with PVT. Additionally, Han et al. ${ }^{9}$ reported that the objective response rate of concurrent HAIC plus radiation therapy for advanced HCC with PVT was 45\% which was significantly higher than that of sorafenib. However, the studies mentioned above are limited by small sample size, retrospective study design, and selection bias. Although the conclusions cannot be generalized, HAIC has a more favorable treatment response than sorafenib. In addition, the therapeutic effect of HAIC has been reported in Japan, and HAIC was shown to have better survival than the control $^{10}$ or TACE groups ${ }^{11}$ in patients with advanced HCC. As shown in this report, HAIC is a good treatment option for advanced HCC patients with PVT in clinical practice.

Our study demonstrates the therapeutic effect of the com-
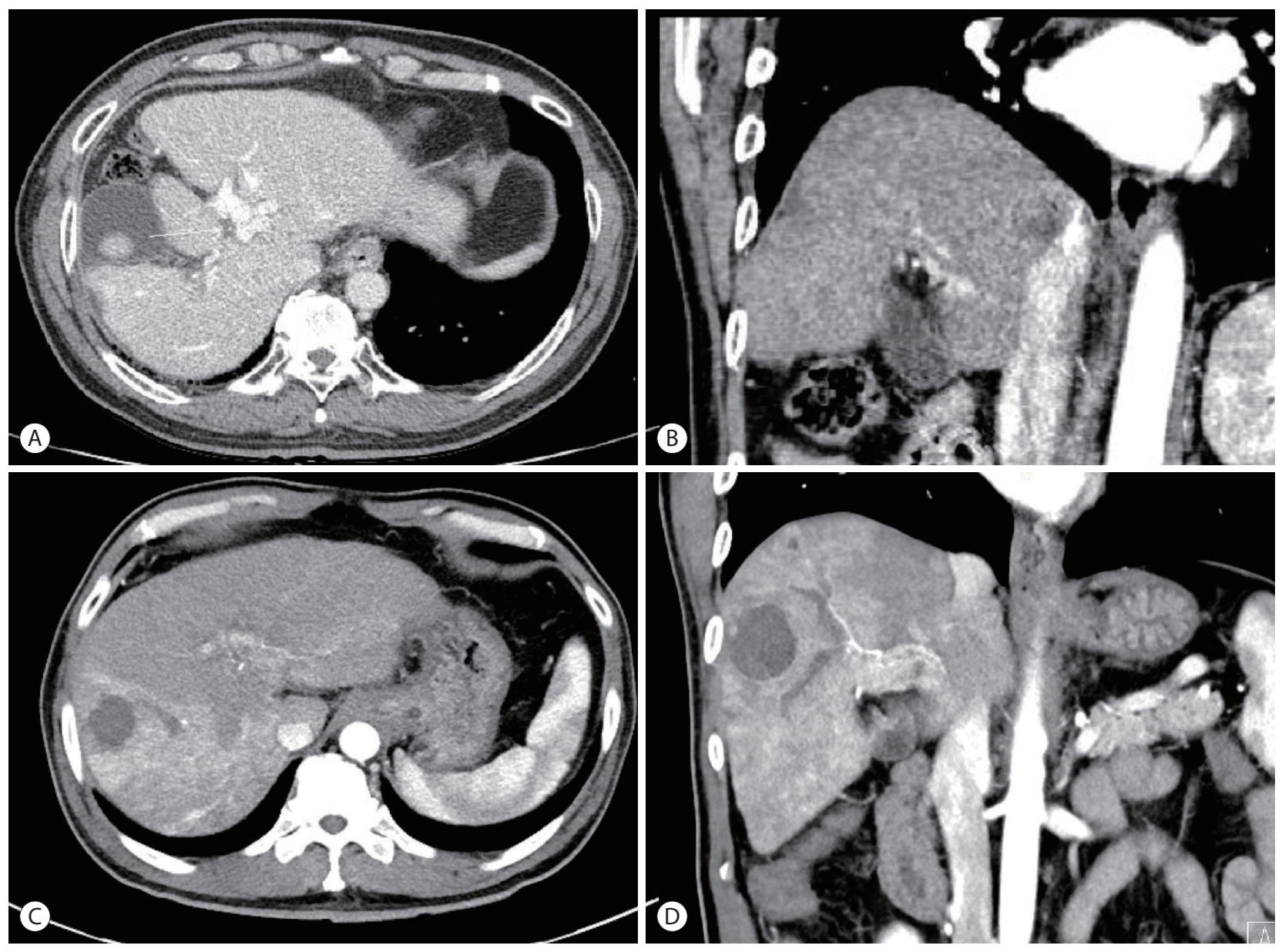

Figure 4. Follow-up computed tomography (CT) finding after 27 months. Follow-up CT imaging was performed 27 months from the first hepatic arterial infusion chemotherapy treatment and 16 months after transarterial chemoembolization. The last follow-up imaging showed a stable state and improved infiltrative hepatocellular carcinoma (HCC) in the right lobe, infarcted HCC in the segment 5/6, and decreased tumor thrombosis in both portal veins (A, B) as compared to the initial CT findings at (C, D). 
bined treatment of HAIC and radiotherapy. The efficacy of the combined treatment of HAIC and radiation in advanced HCC patients with PVT has not been well studied. Zhao et al. ${ }^{12}$ showed that HAIC combined with radiotherapy had significantly better OS than HAIC alone. Therefore, in our center, a combination of HAIC plus radiation therapy is administered in highly selective patients with PVT. However, previous reports showed that this combination therapy was associated with a significant increase in the risk of adverse events such as leukopenia and thrombocytopenia; thus, clinical attention is required. ${ }^{12}$ Similarly, in our study, duodenal ulcer bleeding occurred after radiation therapy, and therefore, radiation treatment was discontinued.

Besides sorafenib, additional treatment modalities are needed for advanced HCC. In this case report, we showed that HAIC might be a viable alternative to sorafenib for patients with advanced HCC with PVT. Further study is needed to determine which patients will benefit from HAIC treatment.

\section{ACKNOWLEDGMENTS}

This work was supported by the Soonchunhyang University Research Fund.

\section{Conflicts of Interest}

The authors have no financial conflicts of interest.

\section{REFERENCES}

1. Forner A, Llovet JM, Bruix J. Hepatocellular carcinoma. Lancet 2012;379:1245-1255.

2. Kwak HW, Park JW, Nam BH, Yu A, Woo SM, Kim TH, et al. Clinical outcomes of a cohort series of patients with hepatocellular carcinoma in a hepatitis B virus-endemic area. J Gastroenterol Hepatol 2014; 29:820-829.
3. Marrero JA, Kulik LM, Sirlin CB, Zhu AX, Finn RS, Abecassis MM, et al. Diagnosis, staging, and management of hepatocellular carcinoma: 2018 Practice Guidance by the American Association for the Study of Liver Diseases. Hepatology 2018;68:723-750.

4. European Association for the Study of the Liver. EASL Clinical Practice Guidelines: management of hepatocellular carcinoma. J Hepatol 2018;69:182-236.

5. Song DS, Song MJ, Bae SH, Chung WJ, Jang JY, Kim YS, et al. A comparative study between sorafenib and hepatic arterial infusion chemotherapy for advanced hepatocellular carcinoma with portal vein tumor thrombosis. J Gastroenterol 2015;50:445-454.

6. Kim SJ, Jang BK, Hwang JS. A case of hepatocellular carcinoma with portal vein tumor thrombosis treated by hepatic artery injection chemotherapy and radiotherapy. J Liver Cancer 2017;17:158162.

7. Saeki I, Yamasaki T, Maeda M, Hisanaga T, Iwamoto T, Matsumoto $T$, et al. Effect of body composition on survival benefit of hepatic arterial infusion chemotherapy for advanced hepatocellular carcinoma: a comparison with sorafenib therapy. PLoS One 2019;14:e0218136.

8. Choi JH, Chung WJ, Bae SH, Song DS, Song MJ, Kim YS, et al. Randomized, prospective, comparative study on the effects and safety of sorafenib vs. hepatic arterial infusion chemotherapy in patients with advanced hepatocellular carcinoma with portal vein tumor thrombosis. Cancer Chemother Pharmacol 2018;82:469-478.

9. Han KH, Seong J, Kim JK, Ahn SH, Lee DY, Chon CY. Pilot clinical trial of localized concurrent chemoradiation therapy for locally advanced hepatocellular carcinoma with portal vein thrombosis. Cancer 2008;113:995-1003.

10. Nouso K, Miyahara K, Uchida D, Kuwaki K, Izumi N, Omata M, et al. Effect of hepatic arterial infusion chemotherapy of 5 -fluorouracil and cisplatin for advanced hepatocellular carcinoma in the Nationwide Survey of Primary Liver Cancer in Japan. Br J Cancer 2013;109:1904-1907.

11. Sumie S, Yamashita F, Ando E, Tanaka M, Yano Y, Fukumori K, et al. Interventional radiology for advanced hepatocellular carcinoma: comparison of hepatic artery infusion chemotherapy and transcatheter arterial lipiodol chemoembolization. AJR Am J Roentgenol 2003;181:1327-1334.

12. Zhao Q, Zhu K, Yue J, Qi Z, Jiang S, Xu X, et al. Comparison of intra-arterial chemoembolization with and without radiotherapy for advanced hepatocellular carcinoma with portal vein tumor thrombosis: a meta-analysis. Ther Clin Risk Manag 2017;13:21-31. 